\title{
A dynamic capacity degradation model and its applications considering varying load for a large format Li-ion battery
}

Minggao OUYANG ${ }^{1, *}$, Xuning FENG ${ }^{1}$, Xuebing $\mathrm{HAN}^{1}$, Languang $\mathrm{LU}^{1}$, Zhe $\mathrm{LI}^{1}$, Xiangming $\mathrm{HE}^{1,2}$

1. State Key Laboratory of Automotive Safety and Energy, Tsinghua University, Beijing 100084, China.

2. Institute of Nuclear and New Energy Technology, Tsinghua University, Beijing, 100084, China.

*Corresponding Author: ouymg@tsinghua.edu.cn

First author E-Mail:fxn07@mails.tsinghua.edu.cn

Address: State Key Laboratory of Automotive Safety and Energy, Tsinghua University, Beijing 100084, China

Tel.: (86)-10-62773437

Fax: (86)-10-62785708 


\section{Abstract}

The capacity degradation of the lithium ion battery should be well predicted during battery system design. Therefore, high-fidelity capacity degradation models that are suitable for the task of capacity prediction are required. This paper proposes a novel capacity degradation model that can simulate the degradation dynamics under varying working conditions for large-format lithium ion batteries. The degradation model is built based on a mechanistic and prognostic model (MPM) whose parameters are closely linked with the degradation mechanisms of lithium ion batteries. Chemical kinetics was set to drive the parameters of the MPM to change as capacity degradation continues. With the dynamic parameters of the MPM, the capacity predicted by the degradation model decreases as the cycle continues. Accelerated aging tests were conducted on three types of commercial lithium ion batteries to calibrate the capacity degradation model. The good fit with the experimental data indicates that the model can capture the degradation mechanisms well for different types of commercial lithium ion batteries. Furthermore, the calibrated model can be used to 1) evaluate the longevity of a battery system under a specific working load and 2) predict the evolution of cell variations within a battery pack when different cell works at different conditions.

Correlated applications are discussed using the calibrated degradation model.

\section{Key words}

Lithium ion battery;

Capacity degradation;

State of health;

Degradation model; 
Chemical kinetics.

\section{Introduction}

Lithium ion batteries have been widely used for electrochemical energy storage, e.g., hybrid electric vehicle [1-4], electric vehicle [5-8], submarine [9], airborne system [10], and electrical energy storage for power grid [11] given their high energy density and extended lifecycle. However, degradation is inevitable during practical use, in other words, the state of health ( $\mathrm{SOH})$ of a lithium ion battery becomes increasingly worse as the cycle continues [12-15]. Generally, a lithium ion battery degrades with a decrease in capacity and increase in resistance $[12,13]$. The capacity decrease and resistance increase directly determine the capability of the power output of the lithium ion battery. Therefore, the degradation of lithium ion batteries should be well predicted during design and accurately estimated during cycling. High-fidelity models are needed for a good prediction of degradation, and on-board $\mathrm{SOH}$ estimation algorithms are needed for an accurate estimation of $\mathrm{SOH}$.

The prediction of degradation is essential when considering the following problems: 1) How long can a lithium ion battery system functions given a specific working load? 2) How will the capacity of a battery pack evolve when single cells work under different conditions [16]? 3) What is the optimal size of a hybrid power system considering the depreciation expense of a lithium ion battery [17]? A high-fidelity capacity degradation model is needed to serve the purpose of prediction. However, such a capacity degradation model for commercial lithium ion batteries is unavailable in the literature to the best knowledge of the authors.

A good degradation model should capture the main degradation mechanisms considering variant aging factors. The main degradation mechanisms of lithium ion batteries include the loss 
of lithium inventory (LLI), the loss of active material (LAM), and the ohmic resistance increase (ORI) [18-20], as shown in Fig. 1. The most influential factors of the capacity degradation includes the temperature, the depth of discharge (DOD), the accumulated ampere hour, the current rate (C-rate), and the SOC [21-23]. Current degradation models can be categorized into the empirical model and the mechanistic model.

The empirical model has capacity as its unique output $[21,24]$. However, the empirical model can only predict the cell capacity without considering correlated degradation mechanisms (LAM and LLI), which may significantly influence the prediction results of the model. The mechanistic model, which can capture the degradation mechanism (LAM and LLI) based on the first principle has higher fidelity than the empirical model.

Mechanistic models are developed based the first principle. Physical-based equations are utilized in the mechanistic models to describe the dynamics of the degradation reactions. For example, the growth of the solid electrolyte interface (SEI) film, one of the widely acknowledged causes of LLI and ORI, has been included in the capacity degradation model, which can interpret the degradation trend of a lithium ion battery [25-28]. Furthermore, the SEI growth coupled with particle cracking has been modeled by Liu et al. in [29]. Christensen and Newman [30,31] proposed a mechanistic and prognostic model (MPM) whose parameters directly link with the LAM, LLI and ORI of a lithium ion battery. The dynamics of LAM, LLI and ORI have been described by Arrhenius Equations, but without experimental validation as in [30,31], because the parameters of the MPM cannot be measured directly. However, Han et al. [32] successfully identified the parameters of the MPM. The identified parameters indicate LAM, LLI and ORI, which conform to the general degradation mechanisms of lithium ion batteries. Arrhenius 
Equations can be used to fit the change of the identified parameters in the MPM, thereby simulating the capacity degradation of lithium ion batteries. However, few previous works have been proposed to fit the parameters with chemical kinetics using Arrhenius Equations. There is still no validated model that can predict the voltage behavior of a lithium ion battery considering all major capacity fading mechanisms (LAM, LLI and ORI) and varying loads (current rate, temperature).

On-board SOH estimation is an active research topic, upon which researchers have provided plenty of effective approaches $[12,13]$. Useful on-board SOH estimation includes extended Kalman filtering [33-35], support vector regression [36,37], probability density function [38], and Delphi method [39] etc. A good on-board SOH estimation algorithm can help improve the estimation accuracy of the battery's state of charge (SOC) and state of function (SOF) [40], thereby benefiting the optimization strategies of the power management for a hybrid electric system [41]. Current on-board estimation algorithms for SOC, SOH or SOF, require a proper update of the open circuit voltage (OCV). A high-fidelity capacity degradation model can serve the purpose of updating the OCV throughout the lifecycle of a lithium ion battery, thereby improving the accuracy of on-board estimation algorithms. However, there are few validated degradation models that can predict the voltage of a lithium ion battery considering capacity fading, to the best knowledge of the authors.

This paper builds a novel capacity degradation model with chemical kinetics considering varying loads for different types of commercial large-format lithium ion batteries. The predicted capacity degradation can fit well with the experimental data. With good prediction of battery voltage considering degradation throughout the whole battery lifecycle, the model can be used to 
update the OCV, which is the critical information for a successful on-board estimation of SOC, $\mathrm{SOH}$, and SOF. Moreover, the model can be used to 1) determine the optimal size of a battery in a hybrid power system; 2) evaluate the longevity of a battery system design under a specific working load; 3) predict the capacity evolution of cell inconsistency within a battery pack when different cells work at different conditions.

\section{Experimental settings}

\subsection{Battery}

Degradation tests were conducted upon three types of commercial lithium ion batteries, of which the specifications are listed in Table 1 . The batteries are assigned with capital characters A, $\mathrm{B}$, and $\mathrm{C}$ for the convenience of further discussion. Two of the batteries have cathodes with $\mathrm{LiFePO}_{4}$ (LFP), whereas the other has a mixed cathode material with $\mathrm{LiMn}_{2} \mathrm{O}_{4}$ (LMO) and $\mathrm{LiNi}_{x} \mathrm{Co}_{y} \mathrm{Mn}_{z} \mathrm{O}_{2}$ (NCM). All batteries have anodes with graphite, marked as "G" in Table 1.

\subsection{Degradation test}

Fig. 2 shows the design of the degradation test. All batteries were fully charged at $1 / 3 \mathrm{C}$, and fully discharged at $1.5 \mathrm{C}$ within one cycle using a Digatron tester inside a thermal chamber. The environmental temperature inside the thermal chamber switches from $45^{\circ} \mathrm{C}\left(5^{\circ} \mathrm{C}\right)$ to $5^{\circ} \mathrm{C}\left(45^{\circ} \mathrm{C}\right)$ after every 90 cycles, as shown in Fig. 2. The reason for selecting this test profile is to simulate the practical working condition of a pure electric vehicle. A temperature of $45^{\circ} \mathrm{C}\left(5^{\circ} \mathrm{C}\right)$ indicates the average working temperature in summer (winter), whereas 90 cycles denotes the half-year usage of the electric vehicle. Readers are referred to [42] for more details on the selection of the test profile.

A reference performance test (RPT) was conducted at $25^{\circ} \mathrm{C}$ using $1 / 3 \mathrm{C}$ current after every 30 
cycles. The capacity measured by the RPT decreases as the cycles proceed, as illustrated by the hollow squares shown in Fig. 2.

\section{Degradation mechanism identification}

\subsection{Capacity loss}

The capacity losses during the degradation test for all batteries are shown in Fig. 3. The capacity retention rates after 990 cycles are $86 \%, 79 \%$ and $60 \%$ for Battery A, B and C, respectively. Generally, when designing a battery pack for an electric vehicle, the battery is considered to reach its end of life (EOL) when the battery capacity degrades to $80 \%$ of its initial value [43-46]. Therefore Battery B (Battery C) reaches its EOL at approximately the $990^{\text {th }}\left(420^{\text {th }}\right.$ ) cycle as shown in Fig. 3. Given that 90 cycles denote a half-year usage of the electric vehicle, the pure electric vehicle with Battery B (Battery C) can expect a normal operation duration of 5.5 years (2.3 years), whereas the pure electric vehicle with Battery A has a longer operation time (more than 6 years).

\subsection{Incremental capacity analysis}

IC analysis is widely used for in situ diagnosis of the battery's SOH [20]. The incremental capacity (IC) curves are the derivatives of the charge/discharge voltage curves. Different peaks in the IC curves indicate different chemical reactions or intercalation processes. The changes in the shape/position of the IC peaks indicate corresponding degradation mechanisms.

IC analyses were conducted to identify the qualitative degradation mechanisms of the Battery A, B and C. Fig. 4 shows the IC curves of Battery A, B and C. Because the IC analyses have already been discussed in [32], here only a brief overview of the results is provided.

For the battery with the LFP/G electrode, the main degradation mechanism is LLI at the 
anode with SEI growth [20,32]. The drop of peak (3), as in Fig. 4(a) and (b), indicate that LLI occurs at the anode for both Battery A and B. Furthermore, the approaching trend of the three peaks for Battery B indicates possible LAM at the anode [20,32]. For a battery with LMO/G electrode, the main degradation mechanism is LAM at the cathode combined with LLI at the anode owing to Mn dissolution [47,48]. The merging of peaks (1), (2) and (3) indicate LAM at the cathode; however, LLI at the anode cannot be determined through the IC curves in Fig. 4(c). Because the IC analysis is restricted to the qualitative analysis, further study is required to quantitatively identify the degradation mechanisms.

\subsection{Parameter identification of the MPM}

A mechanistic and prognostic model (MPM) is exploited to quantify the degradation mechanisms, as performed by Dubarry et al. in [20] with reference made to [30,31,49-52]. The MPM is built based on Eqn. (1), referring to [32].

$$
V_{\text {simu }}=V_{c a}(y)-V_{a n}(x)-I \cdot R
$$

The voltage in the MPM, $V_{\text {simu }}$, is the difference of the cathode potential $V_{c a}(y)$ and the anode potential $V_{a n}(x)$ with an additional potential loss of $I \cdot R$, as illustrated in Fig. 5. The current $I>0$ denotes a discharge process. $R$ is the average ohmic resistance of the battery without considering the variation of SOC for the convenience of the parameter identification of the MPM. $V_{c a}(y)$ varies with $y$, whereas $V_{a n}(x)$ varies with $x$, and the functional relations are acquired from the material properties of the cathode and the anode, respectively. Half cells were assembled with cathode/Li and anode/Li to acquire the $V_{c a}(y)$ and $V_{a n}(x)$, respectively. The $x(y)$ here represents the state-of-charge of the half-cell, i.e. the state-of-charge of the anode (cathode). Because $R$ is the average ohmic resistance without considering the variation of SOC, polarizations at variant SOCs 
are superimposed on the functional relations $V_{c a}(y)$ and $V_{a n}(x)$ to diminish the error of the parameter identification, as reported in [49].

The stoichiometric coefficients $x$ and $y$ change proportionally to the current $I$, according to Eqn. (2) and (3), where $Q_{a n}$ and $Q_{c a}$ are the capacities of the anode and cathode, respectively, whereas $x_{0}$ and $y_{0}$ denote the initial value for $x(t=0)$ and $y(t=0)$.

$$
\begin{aligned}
& x=x_{0}+\int_{0}^{t}\left(I / Q_{a n}\right) d \tau \\
& y=y_{0}-\int_{0}^{t}\left(I / Q_{c a}\right) d \tau
\end{aligned}
$$

The MPM is completely defined using Eqn. (1)-(3). With a proper set of parameters $\left\{Q_{c a}, Q_{a n}\right.$, $\left.y_{0}, x_{0}, R\right\}$, the MPM can achieve a good fit with the discharge curve of the RPT test, as shown in Fig. 5. $\left\{Q_{c a}, Q_{a n}, y_{0}, x_{0}, R\right\}$ change with the degradation process and are directly linked with LLI, LAM, and ORI. For instance, for the discharge process, considering the decrease of $Q_{c a}$ and $Q_{a n}$ indicates LAM at the cathode and anode, respectively; the decrease of $x_{0}$ indicates LLI at the anode; and the increase of $R$ denotes ORI. Therefore, a proper set of $\left\{Q_{c a}, Q_{a n}, y_{0}, x_{0}, R\right\}$, with which the MPM can fit the discharge curve in the RPT test well, can reflect the SOH of the battery.

The proper set of $\left\{Q_{c a}, Q_{a n}, y_{0}, x_{0}, R\right\}$ must be identified through the data recorded in the RPT test. From the RPT test, we can acquire a set of $\{t, V, I\}$, where $t$ is the time, $V$ is voltage measured during the discharge process, and $I=1 / 3 \mathrm{C}$ is the current. The optimization algorithm, i.e., genetic algorithm (GA) has been used to identify the optimal set of parameters $\left\{Q_{c a}, Q_{a n}, y_{0}, x_{0}, R\right\}$ for the model to fit the experimental data $\{t, V(t), I\}$, as Han did in [32]. The fitness function used for the optimization algorithm is $R M S E$ the root mean square error between the voltage in the simulation $\left(V_{\text {Simu }}(t)\right)$ and the voltage in the experiment $\left(V_{\text {Exp }}(t)\right)$ defined in Eqn. (4). The optimization 
algorithm uses RMSE to iterate and find an optimal solution. Note that Eqn. (4) is a discrete expression because the sampling interval during discharge is $1 \mathrm{~s} . T$ is the total time taken in a discharge process.

$$
R M S E=\sqrt{\frac{1}{T} \sum_{t=1}^{T}\left(V_{\text {Simu }}(t)-V_{\text {Exp }}(t)\right)^{2}}
$$

Fig. 6 illustrates the identified optimal sets of $\left\{Q_{c a}, Q_{a n}, y_{0}, x_{0}, R\right\}$ and the $R M S E$ for all batteries during the whole degradation test. The MPM with the identified parameters can fit well with the experimental result throughout the whole test cycle, as shown in Fig. 7. The good fit with the experimental data indicates that the MPM using the identified parameters can capture the degradation mechanisms well. Therefore, the identified parameters can be used as the indicators of the LLI, LAM, and ORI.

\subsection{Degradation mechanism}

Correlated LLI, LAM and ORI can be clearly observed through the identified set of $\left\{Q_{c a}, Q_{a n}\right.$, $\left.y_{0}, x_{0}, R\right\}$, as shown in Fig. 6. The degradation mechanisms of the tested batteries have been summarized in Table 2. The ORI and LLI occur in all batteries, the LAM at the anode exists in Battery B and C; and the LAM at the cathode occurs only in Battery C. The parameter identification provides a possibility for building a capacity degradation model with chemical kinetics that can capture the degradation trends of the parameters. The capacity degradation model is described in Sec. IV.

\section{Degradation modeling}

Dynamic models with chemical kinetics are built to predict the capacity degradation behaviors of the three types of commercial lithium-ion batteries.

\subsection{MPM with degradation kinetics}


The MPM can track the degradation process given that its parameters $\left\{Q_{c a}, Q_{a n}, y_{0}, x_{0}, R\right\}$ can follow the behavior of the identified values in Fig. 6. The MPM in Eqn. (1)-(3) is revised into the degradation model with chemical kinetics applied to describe the change of $\left\{Q_{c a}, Q_{a n}, y_{0}, x_{0}, R\right\}$, as in Eqn. (1), (5)-(16).

The voltage, $V_{\text {simu }}$, is the difference between the cathode potential $V_{c a}(y)$ and the anode potential $V_{a n}(x)$ with an additional potential loss of $I \cdot R$, as presented by Eqn. (1) and shown in Fig.

5.

LLI and LAM at the anode influence the calculation of $x(t)$ in the model, as described in Eqn.

(5). Compared with the original model in Eqn. (2), LLI is considered in the model by adding a depleting term $\Delta x$ in Eqn. (5). $x_{0}$, the initial value of $x$ at the initiation of discharge, is influenced by LLI and can thus be calculated by Eqn. (6), where $x_{0,0}$ denotes $x_{0}$ at the very beginning of cycling, $\Delta x$ is the accumulated loss of $x$ due to LLI, $\int_{0}^{T_{c o c}} \frac{I}{c_{Q, a n} \cdot Q_{a n, 0}} d \tau$ is the integration caused by cycling, and $T_{\text {cyc }}$ is the time elapsed during cycling. $\Delta x$ is calculated by Eqn. (7), where $k_{\mathrm{LLI}}$ denotes the decreasing rate of $x$ due to LLI.

$$
\begin{gathered}
x=x_{0,0}-\Delta x+\int_{0}^{t} \frac{I}{c_{Q, a n} \cdot Q_{a n, 0}} d \tau \\
x_{0}=x_{0,0}-\left.\Delta x\right|_{t=T_{c y c}}+\int_{0}^{T_{a v c}} \frac{I}{c_{Q, a n} \cdot Q_{a n, 0}} d \tau \\
\Delta x=\int_{0}^{t} k_{L L I} d \tau
\end{gathered}
$$

The LAM at the anode is considered in the model by substituting the anode capacity $Q_{\text {an }}$ into the product of $c_{Q, \text { an }}$ and $Q_{\mathrm{an}, 0}$ in Eqn. (5) compared with Eqn. (2). $Q_{\mathrm{an}}$ is assumed to be the product of $c_{Q, \text { an }}$ and $Q_{\mathrm{an}, 0}$ as in Eqn. (8), where $c_{Q, \text { an }}$ indicates the normalized anode capacity, and $Q_{\mathrm{an}, 0}$ is the initial capacity of the anode. $c_{Q \text {,an }}$ decreases because of LAM and can be calculated by Eqn. (9), 
where $c_{Q, \text { an }, 0}=1$ denotes that the initial anode capacity is $100 \%$, and $k_{\mathrm{LAM}, \text { an }}$ denotes the decreasing rate of $c_{Q, \text { an }}$ due to LAM.

$$
\begin{gathered}
Q_{a n}=c_{Q, a n} \cdot Q_{a n, 0} \\
c_{Q, a n}=c_{Q, a n, 0}-\int_{0}^{t} k_{L A M, a n} d \tau
\end{gathered}
$$

The LAM at the cathode influences the calculation of $y(t)$ in the model, as described in Eqn. (10), where $y_{0,0}$ is the stoichiometric coefficient of the cathode at the very beginning of cycling. The LAM at the cathode is considered in the model by substituting the cathode capacity $Q_{\text {ca }}$ into the product of $c_{Q, \mathrm{ca}}$ and $Q_{\mathrm{ca}, 0}$ as shown in Eqn. (10) compared with Eqn. (3). $Q_{\mathrm{ca}}$ is assumed to be the product of $c_{Q, \text { ca }}$ and $Q_{\text {ca, } 0}$ as in Eqn. (11), where $c_{Q, \text { ca }}$ indicates the normalized cathode capacity, and $Q_{\mathrm{ca}, 0}$ is the initial capacity of the cathode. $c_{Q, \text { ca }}$ decreases because of LAM at the cathode, and can be calculated by Eqn. (12), where $c_{Q, \mathrm{ca}, 0}=1$ denotes that the initial anode capacity is $100 \%$, and $k_{\text {LAM,ca }}$ denotes the decreasing rate of $c_{Q, \text { ca }}$ due to LAM. $y_{0}$, the initial value at the start of discharge, changes slightly as the cycle continues, as calculated by Eqn. (13), where $y_{0,0}$ denotes $y_{0}$ at the very beginning of cycling, $\int_{0}^{T_{o c}} \frac{I}{c_{Q, c a} \cdot Q_{c a, 0}} d \tau$ is the integration caused by cycling, and $T_{\mathrm{cyc}}$ is the time elapsed during cycling.

$$
\begin{gathered}
y=y_{0,0}-\int_{0}^{t} \frac{I}{c_{Q, c a} \cdot Q_{c a, 0}} d \tau \\
Q_{c a}=c_{Q, c a} \cdot Q_{c a, 0} \\
c_{Q, c a}=c_{Q, c a, 0}-\int_{0}^{t} k_{L A M, c a} d \tau \\
y_{0}=y_{0,0}-\int_{0}^{T_{c c}} \frac{I}{c_{Q, c a} \cdot Q_{c a, 0}} d \tau
\end{gathered}
$$

ORI is considered in the model using Eqn. (14), where $R_{0}$ is the initial resistance of the battery, $k_{R}$ denotes the growth rate of the ohmic resistance, and $\xi(T)$ is a look-up table function that regulates the resistance at different temperatures as listed in Table 3. Referring to [27,28], the ORI 
is assumed to be caused by the growth of SEI film, of which the growth rate is proportional to the LLI. Therefore, $k_{R}$ is assumed to be proportional to $k_{\mathrm{LLI}}$ as in Eqn. (15), where $K_{\mathrm{SEI}}$ is the gain factor that describes the proportional relationship between ORI and LLI as in [27,28]. The values of $K_{\mathrm{SEI}}$ for different batteries in the model have been listed in Table 3.

$$
\begin{gathered}
R=\left(R_{0}+\int_{0}^{t} k_{R} d t\right) \cdot \xi(T) \\
k_{R}=K_{S E I} \cdot k_{L L I}
\end{gathered}
$$

Both LLI and LAM are caused by side reactions [32,47], of which the reaction rates can be described by the chemical kinetics. The Arrhenius Equations, which is universally acknowledged in chemical kinetics, can be utilized to calculate the degradation rates ( $k_{\mathrm{LLI}}, k_{\mathrm{LAM}, \text { an }}$ and $\left.k_{\mathrm{LAM}, \mathrm{ca}}\right)$ as in Eqn. (16). To best capture the degradation behavior under different influential factors, the Arrhenius Equation is revised into the form of Eqn. (16) according to the modeling work in [21].

$$
k_{\beta}=\left\{\begin{array}{lr}
-A_{\beta} \cdot c_{\beta}^{n} \cdot \exp \left(-\frac{E_{a, \beta}-K_{\beta} \cdot\left|C_{R a t e}\right|^{m}}{R T}\right) \cdot f(\beta), & \text { (if LLI or LAM exists) } \\
0, & \text { (if no LLI or LAM exists) }
\end{array}\right.
$$

The Arrhenius Equation in Eqn. (16) includes most of the aging factors. $T$ denotes the temperature during cycling, and a higher $T$ results in a faster degradation. $\left|C_{\text {rate }}\right|$ is the absolute value of the current rate, and a larger $\left|C_{\text {rate }}\right|$ leads to a faster degradation. The frequency factor $\left(A_{\beta}\right)$ and the activation energy $\left(E_{a, \beta}\right)$ cooperate to determine the reaction rate $k_{\beta}$. The subscript $\beta$ is a general index, which can be "LLI", "LAM, an" or "LAM,ca" to represent the degradation reaction of LLI or LAM at the anode or cathode. $c_{\beta}$ denotes the normalized concentration of the reactants for reaction $\beta$, as listed in Table 4 . When simulating the LLI at the anode, $c_{\beta}$ equals $x$ which varies with the SOC of the battery; therefore the influence of SOC on the capacity degradation is also considered in the model. 
In Eqn. (16), $n$ and $m$ are the reaction orders for the Arrhenius Equation. $n$, no less than 1, reflects the influence of the normalized concentration $c_{\beta}$ on the reaction rates. A larger $n$ leads to a much slower reaction rate when $c_{\beta}$ is small, and the reaction thus decelerates as the degradation continues given $n>1$. $m$, usually no more than 1 , reflects the influence of $C_{\text {Rate }}$ on the degradation. A smaller $m$ denotes a smaller influence of $C_{\text {Rate }}$ on the capacity degradation rates. When $m=0$, there is no influence on the capacity degradation caused by $C_{\text {Rate }}$, and the integrated degradation behavior can be described using the total Ah throughput.

To well fit the identified parameters $\left\{Q_{c a}, Q_{a n}, y_{0}, x_{0}, R\right\}$ in Fig. 6, the capacity degradation mechanisms listed in Table 2 are applied. The correlated parameters in Eqn. (16) were calibrated as listed in Table 5 .

\subsection{Validation of the degradation model}

The degradation model has been calibrated using the parameters listed in Table 5. The parameters in the degradation model with the chemical kinetics (in Eqn. (16)) can track the degradation trends of the identified parameters (in Fig. 6), as shown in Fig. 8. The model is cycled using the RPT profile depicted in Sec 2.2, and the predicted capacity of the degradation model can fit well with the depleting capacity measured in the experiments. Moreover, the degradation model can also fit the charge/discharge voltage curves of the batteries at different SOHs, given that the parameters in the degradation model can capture the degradation behavior of the identified parameters, as shown in Fig. 9. The prediction error, which is the difference of the predicted voltage and the voltage in experiment have been listed using Fig. 10. The predicted voltage at middle range of SOC (10\% 90\%), both for the charge and discharge curve, are quite close to the voltage in experiment. However, for the charging process at SOC $<10 \%$ and the discharging process at $\mathrm{SOC}>90 \%$, the predicted voltage can have an error up to $0.1 \sim 0.4 \mathrm{~V}$. In the MPM, the 
resistance $\mathrm{R}$ is considered as a lumped parameter through all values of SOC, whereas in practical condition, the battery has high resistance at SOC near $0 \%$ and $100 \%$. Therefore the large error at low SOC for charging and high SOC for discharging is inevitable without changing the lumped resistance $\mathrm{R}$ in the degradation model. The calibration of $\mathrm{R}$ considering variant $\mathrm{SOC}$ can be further conducted referring to $[49,53]$. However, we do not discuss it because related discussions seem to be trivial.

All in all, the good fit of the degradation features in the experiment lead us to believe that the degradation model has high fidelity to predict the degradation behavior of the battery.

A further case helps convince us of the reliability of the degradation model. From the user manual provided by the manufacturer, Battery A reaches its EOL after 2500-3000 cycles under 1C charge/discharge at $25^{\circ} \mathrm{C}$. Under the same test profile, the degradation model for Battery A predicts that the battery will reach its EOL after 3000 cycles, which conforms to the reference number provided by the manufacturer. The capacity degradation of the model for Battery A is shown in Fig. 11(a), with correlated LLI and ORI shown in Fig. 11(b) and (c), respectively. In addition, Battery B (C) reaches its EOL after approximately 1800 (750) cycles as predicted by the degradation model.

\subsection{Computing time of the degradation model}

The proposed degradation model has been simulated in Matlab Simulink $8.3^{\circledR}$ using a laptop with a CPU of Intel ${ }^{\circledR}$ Core $^{\mathrm{TM}}$ i7-3630QM @ 2.4GHz. The computing time for 1000 cycles of the capacity degradation with a time step of $1 \mathrm{~s}$ is about $15 \mathrm{~min}$, which is quite small, because 1000 cycles take at least 2000 hours during practical experiment.

\section{Model applications}

The applications of the degradation model include: (1) determine the optimal size of the 
lithium ion battery in a hybrid power system; (2) evaluate the longevity of a battery system design under a specific working load; (3) predict the evolution of cell inconsistency within a battery pack when different cells work at different conditions. Application (1) has already been discussed in [17,54], readers are referred to [17,54] for detailed information on this type of application. Applications (2) and (3) are discussed as follows.

\subsection{Life prediction given specific working profile}

The degradation model proposed in this paper can be used to evaluate the longevity of a battery that is exploited under a given working profile. A practical example is provided below.

An energy storage system (ESS) is essential for the frequency regulation of a power grid. When the power grid operators are selecting the energy storage system (ESS) for a grid, the lifecycle of the ESS must be evaluated considering the frequency regulation task. Generally, the lifecycle of the ESS should be longer than 10 years. Whether the selected lithium ion battery is able to work normally for 10 years could be conveniently evaluated by the degradation model.

The degradation model for Battery A was simulated to judge whether Battery A can be functional for the requirement of the grid service. The PNNL cycle, which is proposed in [55], is selected as the charge/discharge condition representing the frequency regulation task, as shown in Fig. 12. The voltage, the degradation rates of LLI $\left(k_{\mathrm{LLI}}\right)$, and the integrated normalized LLI at the anode $\left(x_{0}\right)$ can be predicted using the degradation model under the PNNL cycle, as shown in Fig. 12. Therefore, the lifecycle of the Battery A for grid use can be evaluated without a ten-year test.

One PNNL cycle includes $24 \mathrm{~h}$ of frequent current fluctuations representing the task of frequency regulation. The lithium ion battery is assumed to work for 60 full PNNL cycles per year. As the LLI accumulates with the on-going cycling, the capacity degrades until it reaches the EOL at $80 \% \mathrm{SOH}$, as shown in Fig. 13. According to the predicted result, Battery A has a longevity of 4 
years for grid use. To match the requirement of the 10-year service, the lifecycle of Battery A under $1 \mathrm{C}$ cycle at $25^{\circ} \mathrm{C}$ should be improved from 3000 cycles to approximately 8000 cycles, which can already be fulfilled by some of the manufacturers, e.g., Lishen in Tianjin, China [56].

\subsection{Evolution of cell variations caused by temperature difference in a battery pack}

A second example comes from a practical application for the pure electric vehicle (EV). When designing a thermal management system for a battery pack, a common situation is that the temperature variation should be confined within $5^{\circ} \mathrm{C}$. However, a temperature variation as high as $5^{\circ} \mathrm{C}$ still exist among the battery cells. The cells working at different temperatures will degrade at different degradation rates, according to Eqn. (16). Therefore, a beneficial discussion the extent to which the cell variations will evolve given a temperature difference of $5^{\circ} \mathrm{C}$ within the battery pack.

Assume that two cells of Battery B work at $25^{\circ} \mathrm{C}$ and $30^{\circ} \mathrm{C}$, with a temperature difference of $5^{\circ} \mathrm{C}$. The two cells of Battery B are connected in series the same as the real connection in an EV. For battery cells connected in series, the end of charge (EOC) is determined by the maximum voltage of the cells, whereas the end of discharge (EOD) is determined by the minimum voltage of the cells, as shown in Fig. 14.

The degradation models of the two cells, which are connected in series, were cycled to investigate the evolution of the cell variations given a temperature difference of $5^{\circ} \mathrm{C}$. The Federal Urban Driving Schedule (FUDS) as reported in [44] and shown in Fig. 15(a) was applied as the working load for the EV. The two cells were cycled by FUDS until the minimum voltage reached the cut-off voltage of $2.5 \mathrm{~V}$, as shown in Fig. 15(b). The battery cells were charged by a constant current of $1 / 3 \mathrm{C}$ to $100 \%$ SOC after the FUDS test. The capacity degradation rate $\left(k_{\mathrm{LLI}}\right)$ and accumulated degradation ( $\left.\Delta x_{0}\right)$ due to LLI are shown in Fig. 15(b). Fig. 15(b) shows that 1) Cell 2 working at a higher temperature $\left(30^{\circ} \mathrm{C}\right)$, fades faster than Cell $\left.1 ; 2\right) k_{\mathrm{LLI}}$ varies with the C-rate and 
the SOC of the battery cell. A higher C-rate or a higher SOC results in a faster LLI.

Assume that the fully discharge/charge as shown in Fig. 15(b) simulates a one-day working condition for the EV; capacity degradation versus time of use is presented in Fig. 16, with correlated LLI, ORI, and LAM presented on the right. According to the simulation results, the battery pack containing two cells with a temperature variation of $5^{\circ} \mathrm{C}$ reaches its $\mathrm{EOL}$ after 600 days. The capacity of the battery pack is determined by Cell 2 cycled at $30^{\circ} \mathrm{C}$ because Cell 2 has a faster capacity degradation rate. When Cell 2 reaches its EOL, Cell 1 still has $81.4 \%$ of its original capacity. However, the EV may not always discharge to its EOD after one-day of use; thus, the lifecycle of the battery pack may be longer than 600 days.

\subsection{Capacity degradation of battery pack considering cell variations}

Furthermore, the degradation model can help investigate the group evolution behavior of cells within a battery pack. Owing to the cell variations, the capacity of the battery pack is not as much as the capacity of any cell [16]. According to Eqn. (17) from [16], the capacity of an $N$-cell battery pack $\left(Q_{\text {Pack }}\right)$ is determined by two cells: one contains the minimum chargeable electric quantity, $\min _{j=1,2, \ldots N}\left\{\left(1-S O C_{j}\right) \cdot Q_{j}\right\}$; one contains the minimum dischargeable electric quantity, $\min _{j=1,2, \ldots N}\left\{S O C_{j} \cdot Q_{j}\right\}$. Taking the chargeable/dischargeable electric quantity as the $y$ axis, and the battery capacity as the $x$ axis, $Q_{\text {Pack }}$ can be conveniently determined through an $E$ - $Q$ diagram as shown in Fig. 17. The pink dot in Fig. 17 denotes the cell with the minimum rechargeable electric quantity, whereas the green dot denotes the cell with the minimum dischargeable electric quantity. $Q_{\text {Pack }}$ is the $x$-value of the red dot, which is the intersection of the pink line and the green line. The grey dots represent the other cells in the battery pack. Moreover, the equal SOC line can be presented in the $E$ - $Q$ diagram, as the blue dash-and-dot line in Fig. 17. 


$$
Q_{\text {Pack }}=\min _{j=1,2, \ldots N}\left\{\left(1-S O C_{j}\right) \cdot Q_{j}\right\}+\min _{j=1,2, \ldots N}\left\{S O C_{j} \cdot Q_{j}\right\}
$$

Individual dots move as capacity degradation occurs; thus, the $Q_{\text {Pack }}$ evolves in Fig. 17. The movements of the dots denote the evolution of the cell variations and thereby influence the decrease of $Q_{\text {Pack. }}$ The moving dynamics can be described by the degradation model calibrated in this paper.

Assume that six cells of Battery $\mathrm{C}$ are connected in series to form a battery pack. The degradation behavior of each cell is simulated by an independent degradation model calibrated in Table 5. To investigate the influence of the cell variation on the capacity degradation of the batter pack, deviations were set in the degradation kinetics for the six cells. The variation in the chemical kinetics is set by Eqn. (18), where $k_{\beta, 0}$ represents the reaction kinetics without considering deviation, $k_{\beta}$ represents the reaction kinetics considering deviation, and $\Delta k_{\beta}$ represents the deviation coefficient that causes the inconsistency in the pack degradation model. A non-zero $\Delta k_{\beta}$, as set in Table 6 for each cell, can be caused by minor deviation during the manufacturing process.

$$
k_{\beta}=k_{\beta, 0} \cdot\left(1+\Delta k_{\beta}\right)
$$

The series-connected battery pack was simulated under the same test profile in Sec. 2.2. Fig. 18 shows the voltage curve for each cell in the RPT test on the six-cell pack after 990 high-low temperature cycles. Obvious cell variations can be observed in Fig. 18 and the pack capacity is determined by Cell 2 (with the minimum dischargeable capacity) and Cell 5 (with the minimum chargeable capacity).

The parameters $\left\{Q_{c a}, Q_{a n}, y_{0}, x_{0}, R\right\}$ evolve with minor deviations (Eqn. (18) and Table 6) in the degradation kinetics (Eqn. (16)) as the cycle continues, as shown in Fig. 19. According to the dynamic deviations set in Table 6, the major variations in the parameters are located at $x_{0}, Q_{\mathrm{ca}}$ and 
$R$. Owing to the cell variations, $Q_{\text {Pack }}$ is smaller than the minimum capacity of the six cells.

Fig. 20 shows the evolution of the degradation in the battery pack and the determination of the pack capacity. Fig. 20(a) displays the cell variations in the $E-Q$ diagram after different cycles at different SOCs. As the cycle increases, the dots converge towards the origin of the $E$ - $Q$ diagram, indicating continuous capacity degradation. The scattering of the dots denote the deterioration in the cell variation within the battery pack. Fig. 20(b) is a magnification of Fig. 20(a), showing the spread-out trend of the cells at the $25 \%$ SOC line. Cell 2 (the green dots) with the minimum dischargeable electric quantity and Cell 5 (the red dots) with the minimum chargeable electric quantity determine the $Q_{\text {Pack}}$, as shown in Fig. 20(b). Dots for other cells are located inside the acute angle of $45^{\circ}$. At the end of the $990^{\text {th }}$ cycle, $Q_{\text {Pack }}=5.8 \mathrm{Ah}$, whereas the minimum capacity of the six cells is $5.85 \mathrm{Ah}$ for Cell 2, indicating that the cell variation causes a capacity loss of 0.05 Ah for the battery pack in this case. Given that the maximum capacity is 6.16 Ah for Cell 3, the maximum capacity variation among the six-cell pack should be $0.31 \mathrm{Ah}$.

The capacity loss caused by the cell variation in this case $(0.05 \mathrm{Ah})$ seems to be minor. However, the result represents only the case in which all cells have the same initial condition (same electric quantity and same capacity). Simulations that investigate the influence of initial cell variation on the evolution of pack capacity can be further conducted based on the degradation model. Furthermore, it should be noted that, in this case, no more than $10 \%$ deviations in kinetics have been set for the LLI at the anode, the LAM at the cathode, or ORI. The influences of temperature, i.e., the LAM at the anode, the columbic coefficient, the self-discharge rate, etc. are not considered in the discussion above. According to the discussion in Sec. 5.2, a temperature difference of $5^{\circ} \mathrm{C}$ can result in a $1.4 \%$ cell variation at the EOL of the battery pack. Kinetic 
deviations for the LAM at the anode can be treated similarly to Eqn. (18) in a further study. The study of the columbic coefficient is ongoing referring to [57], whereas the study on the self-discharge rate remains uncertain and requires further investigation [58].

\section{Discussions and conclusions}

A capacity degradation model with chemical kinetics considering a varying load for a large format lithium ion battery is built in this paper. Accelerated aging tests were conducted on three types of commercial lithium ion batteries. Correlated degradation mechanisms have been analyzed by identifying the parameters in the MPM. The good fit with the experimental data indicates that the MPM using the identified parameters can capture the degradation behavior well. Therefore the identified parameters can be used as indicators of the LLI, LAM and ORI.

The Arrhenius Equation has been utilized to calculate the degradation rates of the parameters in the MPM. The Arrhenius Equation can capture the degradation behaviors of the identified parameters considering various influential factors. The degradation model with calibrated Arrhenius Equations can track the degradation trend of the identified parameters and thus fit the charge/discharge curve well with the experiment data. The good fit of the degradation and the experimental data indicates that the degradation model has high fidelity to predict the degradation behavior of a battery.

Correlated applications of the degradation model were presented in Sec. 5. First, the lifecycle of a lithium ion battery under a specific working load is evaluated through the degradation model. The results of the degradation model suggests that the lifecycle of Battery A must be further extended to satisfy the ten-year usage for the grid. Second, the evolution of cell variations caused by a temperature difference of $5^{\circ} \mathrm{C}$ in a battery pack for a pure $\mathrm{EV}$ is discussed. The battery pack with Battery B can cycle for approximately 600 days before its EOL. Cell variation of $1.4 \%$ at the 
EOL is caused by the $5^{\circ} \mathrm{C}$-temperature difference. Third, the capacity degradation of a six-cell battery pack considering cell variations is investigated using the degradation model. The evolution of the cell variations due to deviations in kinetics is studied. The result suggests that the pack capacity might be quite close to the cell with minimum capacity, given that all cells start with the same initial status.

Further research includes: 1) designing a more detailed test matrix for degradations tests to calibrate the parameters in Eqn. (16) more precisely and 2) applying the degradation model to solve the degradation-related problem in the battery system design.

\section{Acknowledgment}

This work was supported by US-China Clean Energy Research Center-Clean Vehicle Consortium (CERC-CVC). The CERC-CVC in China side is supported by the MOST (Ministry of Science and Technology) of China under the contract of No. 2014DFG71590, and in U.S. side supported by the Department of Energy under Award Number DE-PI0000012.

Appreciations should be given to Prof. Huei Peng, Prof. Jing Sun and Dr. Caihao Weng with University of Michigan, Ann Arbor for their beneficial advices. The first author appreciates the funding from China Scholarship Council.

\section{References}

[1] Zou Y, Sun F, Hu X, Guzzella L, Peng H. Combined optimal sizing and control for a hybrid tracked vehicle. Energies, 2012, 5: 4697-4710.

[2] Kim MJ, Peng H. Power management and design optimization of fuel cell/battery hybrid vehicles. J. Power Sources, 2007, 165: 819-832.

[3] Feng X, Li J, Lu L, et al. Research on a battery test profile based on road test data from hybrid fuel cell buses. J. Power Sources, 2012, 209: 30-39. 
[4] Zheng Y, Ouyang M, Lu L, et al. Cell state-of-charge inconsistency estimation for $\mathrm{LiFePO}_{4}$ battery pack in hybrid electric vehicles using mean-difference model. Applied Energy, 2013, 111: $571-580$.

[5] Marongiu A, Marco R, Sauer D U. Influence of the vehicle-to-grid strategy on the aging behavior of lithium battery electric vehicles. Applied Energy, 2015, 137: 899-912.

[6] Zheng Y, Han X, Lu L, et al. Lithium ion battery pack power fade fault identification based on Shannon entropy in electric vehicles. J. Power Sources, 2013, 223: 136-146.

[7] Xiong R, Sun F, Chen Z, He H. A data-driven multi-scale extended Kalman filtering based parameter and state estimation approach of lithium-ion polymer battery in electric vehicles. Applied Energy, 2014, 113: 463-476.

[8] Wang Y, Zhang C, Chen Z, Xie J, Zhang X. A novel active equalization method for lithium-ion batteries in electric vehicles. Applied Energy, 2015, 145: 36-42.

[9] Mohiuddin H, Morsalin S, Mahmud K. Design and fabrication of a prototype submarine using Archimedes principle. $3^{\text {rd }}$ International Conference on Informatics, Electronics \& Vision (ICIEV), 2014, pp. 1-6.

[10] Becer J, Schaeper C, Muennix C, Sauer D U. Design of a safe and reliable Li-ion battery system for applications in airborne system. In 52 ${ }^{\text {nd }}$ Aerospace Sciences Meeting, 2014.

[11] Leadbetter J, Swan L G. Selectin of battery technology to support grid-integrated renewable electricity. J. Power Sources, 2012, 216: 376-386.

[12] Lu L, Han X, Li J,et al. A review on the key issues for lithium-ion battery management in electric vehicles. J. Power Sources, 2013, 226: 272-288.

[13] Farmann A, Waag W, Marongiu A, Sauer D U. Critical review of on-board capacity estimation techniques for lithium-ion batteries in electric and hybrid electric vehicles. J. Power Sources, 2015, 281: 114-130.

[14] Waag W, Fleischer C, Sauer D U. Critical review of the methods for monitoring of lithium-ion batteries in electric and hybrid vehicles. J. Power Sources, 2014, 258: 321-339.

[15] Ecker M, Gerschler J B, Vogel J, Kabitz S, Hust F, Dechent P, Sauer D U. Development of a lifetime prediction model for lithium-ion batteries based on extended accelerated aging test data. J. 
Power Sources, 2012, 215: 248-257.

[16] Zheng Y, Ouyang M, Lu L, Li J. Understanding aging mechanisms in lithium-ion battery packs: From cell capacity loss to pack capacity evolution. J. Power Sources, 2015, 278: 287-295.

[17] Hou C, Wang H, Ouyang M. Battery size in for plug-in hybrid electric vehicles in Beijing: A TCO model based analysis. Energies, 2014, 7: 5374-5399.

[18] Zhang Q, White R E. Calendar life study of Li-ion pouch cells. J. Power Sources, 2007, 173: 990-997.

[19] Zhang Q, White R E. Calendar life study of Li-ion pouch cells, Part 2: Simulation. J. Power Sources, 2008, 179: 785-792.

[20] Dubarry M, Truchot C, Liaw B Y. Synthesize battery degradation modes via a diagnostic and prognostic model. J. Power Sources, 2012, 219: 204-216.

[21] Wang J, Liu P, Hicks-Garner, et al. Cycle-life model for graphite-LiFePO 4 cells. J. Power Sources, 2011, 196: 3942-3948.

[22] Li Z, Lu L, Ouyang M, Xiao Y. Modeling the capacity degradation of LiFePO4/graphite batteries based on stress coupling analysis. J. Power Sources, 2011, 196: 9757-9766.

[23] Waag W, Kaebitz S, Sauer D U. Experimental investigation of the lithium-ion battery impedance characteristic at various conditions and aging states and its influence on the application. Applied Energy, 2013, 102: 885-897.

[24] Bloom I, Cole B W, Sohn J J, et al. An accelerated calendar and cycle life study of Li-ion cells. J. Power Sources, 2001, 101: 238-247.

[25] Spotnitz R. Simulation of capacity fade in lithium-ion batteries. J. Power Sources, 2003, 113: $72-80$.

[26] Broussely M, Biensan Ph, Bonhomme F et al. Main aging mechanisms in Li ion batteries. J. Power Sources, 2005, 146: 90-96.

[27] Ramadass P, Haran B, Gomadam P M, White R E, Popov B N. Development of first principles capacity fade model for Li-ion cells. J. Electrochem. Soc., 2004, 151(2): A196-A203.

[28] Ning G, White R E, Popov B N. A generalized cycle life model of rechargeable Li-ion batteries. Electrochimica Acta, 2006, 51: 2012-2022. 
[29] Deshpande R, Verbrugge M, Cheng Y T, et al. Battery cycle life prediction with coupled chemical degradation and fatigue mechanics. J. Electrochem. Soc., 2012, 159 (10): A1730-A1738. [30] Christensen J, Newman J. Effect of anode film resistance on the charge/discharge capacity of a lithium-ion battery. J. Electrochem. Soc. 2003, 105(11): A1416-A1420.

[31] Christensen J, Newman J. Cyclable lithium and capacity loss in Li-ion cells. J. Electrochem. Soc., 2005, 152(4):A818-A829.

[32] Han X, Ouyang M, Lu L, et al. A comparative study of commercial lithium ion battery cycle life in electric vehicle: Aging mechanism identification. J. Power Sources, 2014, 251: 38-54.

[33] Plett G L. Extended Kalman filtering for battery management systems of LiPB-based HEV battery packs, Part 3. State and parameter estimation. J. Power Sources, 2004, 134: 277-292.

[34] Bai G, Wang P, Hu C, Pecht M. A generic model-free approach for lithium-ion battery health management. Applied Energy, 2014,135: 247-260.

[35] Hu C, Youn B D, Chung J. A multiscale framework with extended Kalman filter for lithium-ion battery SOC and capacity estimation. Applied Energy, 2012, 92: 694-704.

[36] Weng C, Cui Y, Sun J, Peng H. On-board state of health monitoring of lithium-ion batteries using incremental capacity analysis with support vector regression. J. Power Sources, 2013, 235: $36-44$.

[37] Weng C, Sun J, Peng H. Model parameterization and adaptation based on the invariance of support vectors with applications to battery state-of-health monitoring. IEEE Trans. on Vehicular Technology, 2015, 64(9): 3908-3917.

[38] Feng X, Li J, Ouyang M, et al. Using probability density function to evaluate the state of health of lithium-ion batteries. J. Power Sources, 2013, 232: 209-218.

[39] Sun B, Jiang J, Zheng F, et al. Practical state of health estimation of power batteries based on Delphi method and grey relational grade analysis. J. Power Sources, 2015, 282: 146-157.

[40] Zou Y, Hu X, Ma H, Li S E. Combined state of charge and state of health estimation over lithium ion battery cell cycle lifespan for electric vehicles. J. Power Sources, 2015, 273: 793-803. 
[41] Masih-Tehrani M, Ha'iri-Yazdi M R, Esfahanian V, Safaei A. Optimum sizing and optimum energy management of a hybrid energy storage system for lithium battery life improvement. J. Power Sources, 2013, 244: 2-10.

[42] Han X, Ouyang M, Lu L, Li J. A comparative study of commercial lithium ion battery cycle life in electric vehicle: Capacity loss estimation. J. Power Sources, 2014, 268: 658-669.

[43] Life cycle testing of electric vehicle battery modules. SAE J2288-1997.

[44]Electric vehicle battery test procedures manual, Rev. 2, USABC, 1996.

[45] Krieger EM, Cannarella J, Arnold CB. A comparison of lead-acid and lithium-based battery behavior and capacity fade in off-grid renewable charging applications. Energy, 2013, 60: 492-500.

[46] Arunachala R, Makinejad K, Athlekar S, Jossen A, Garche J. Cycle life characterisation of large format Lithium-ion cells. in EVS-27.

[47] Zhan C, Lu J, Kropf AJ, et al. Mn(II) deposition on anodes and its effects on capacity fade in spinel lithium manganate-carbon systems. Nature communications, 4(2437): 1-8.

[48] Dai Y, Cai L, White RE. Capacity fade model for spinel $\mathrm{LiMn}_{2} \mathrm{O}_{4}$ electrode. J. Electrochem. Soc., 2013, 160(1): A182-A190.

[49] Albertus P, Couts J, Srinivasan V, Newman J. A combined model for determining capacity usage and battery size for hybrid and plug-in hybrid electric vehicles. J. Power Sources, 2008, 183: 771-782.

[50] Hokura K, Takahashi K, Horiba T. Capacity fading prediction of lithium-ion batteries based on discharge curve analysis. J. Power Sources, 2011, 196: 10141-10147.

[51] Dahn HM, Smith AJ, Burns JC, Stevens DA, Dahn JR. User-friendly differential voltage analysis freeware for the analysis of degradation mechanisms in Li-ion batteries. J. Electrochem. Soc. 2012, 159(9): A1405-A1409.

[52] Bloom I, Walker LK, Basco JK, Abraham DP, Christophersen JP, Ho CD. Differential voltage analyses of high-power lithium-ion cells: 4. Cells containing NMC. J. Power Sources, 2010, 195: $877-882$.

[53] Dubarry M, Vuillaume N, Liaw BY. From single cell model to battery pack simulation for 
Li-ion batteries. J. Power Sources, 2009, 186: 500-507.

[54] Song Z, Hofmann H, Li J, et al. Optimization for a hybrid energy storage system in electric vehicles using dynamic programming approach. Applied Energy, 2015, 139: 151-162.

[55] Protocol for uniformly measuring and expressing the performance of energy storage systems. PNNL-22010, Oct. 2012.

[56] Private communications with Jae Woong Kim, who is the vice president of Tianjin Lishen Battery Joint-stock Co. Ltd.

[57] Zheng Y, Ouyang M, Lu L, et al. Study on the correlation between state of charge and coulombic efficiency for commercial lithium ion batteries. J. Power Sources, 2015, 289: 81-90.

[58] Zheng Y. Study on cell variations of lithium ion power battery packs in electric vehicles. $\mathrm{Ph}$.

D. Thesis Dessertation, Tsinghua Univ., Beijing, June 2014. 


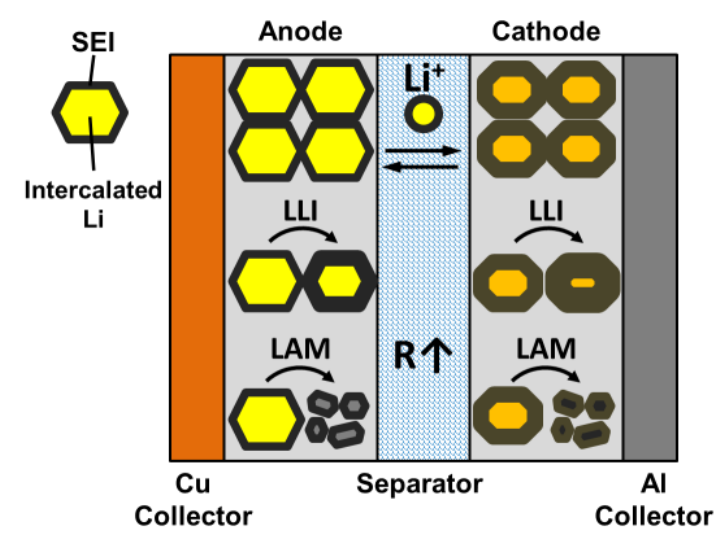

Figure 1 Typical degradation mechanisms of Li-ion battery

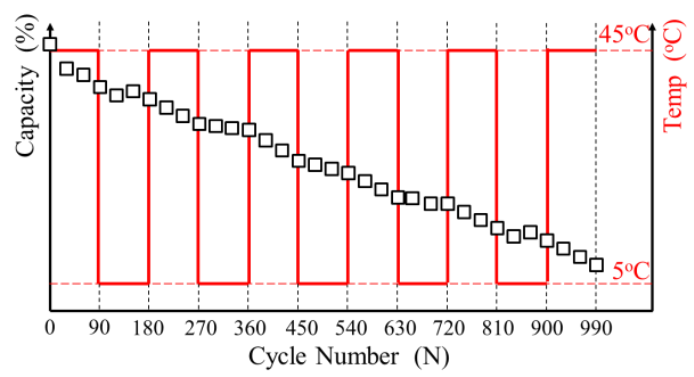

Figure 2 The design of the degradation test.

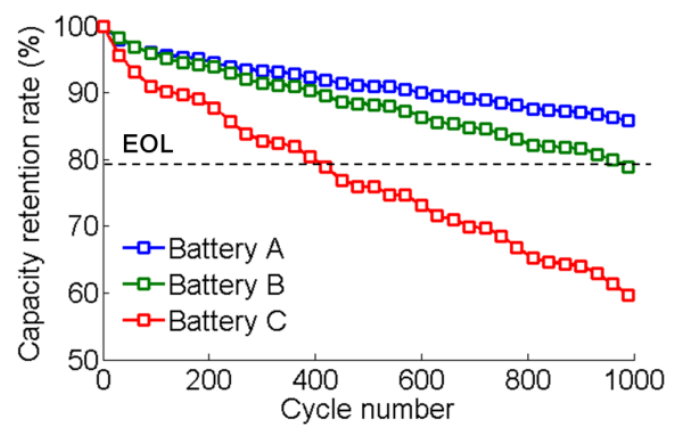

Figure 3 Capacity retention rate during aging test.
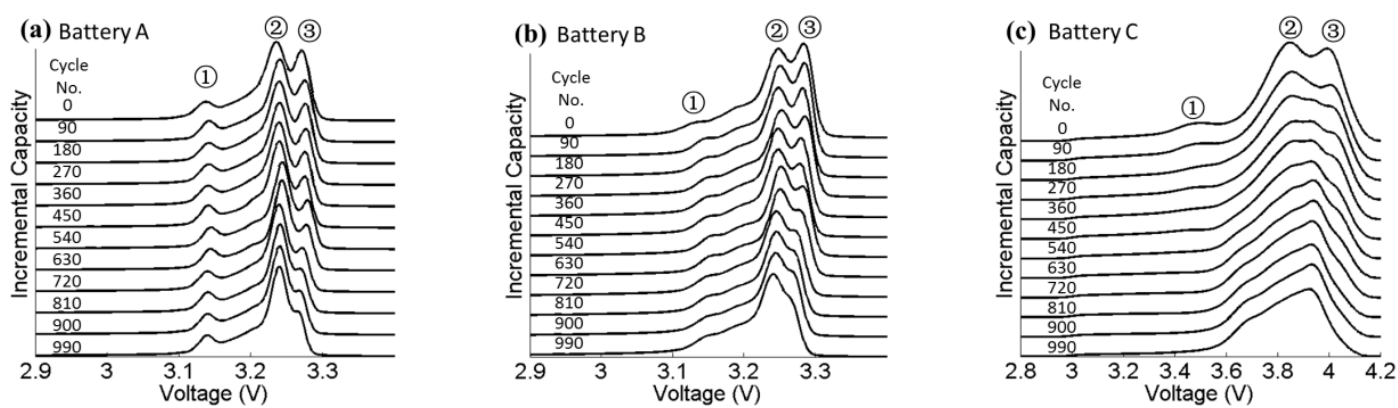

Figure 4 IC curves for degradation mechanism analysis. (a) Battery A; (b) Battery B; (c) Battery C. 


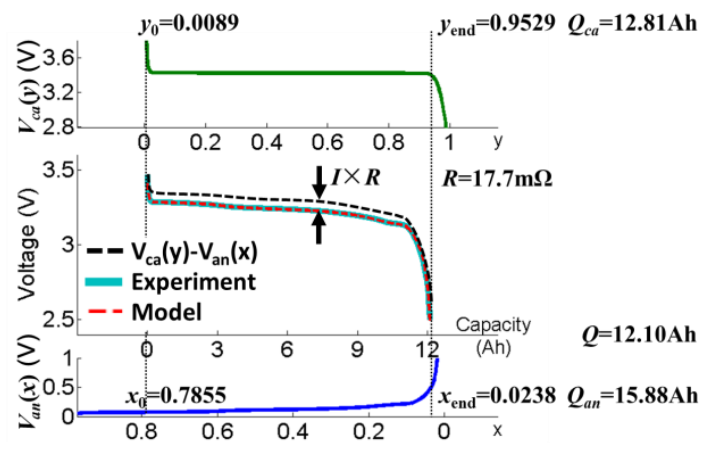

Figure 5 Illustration of the MPM model.
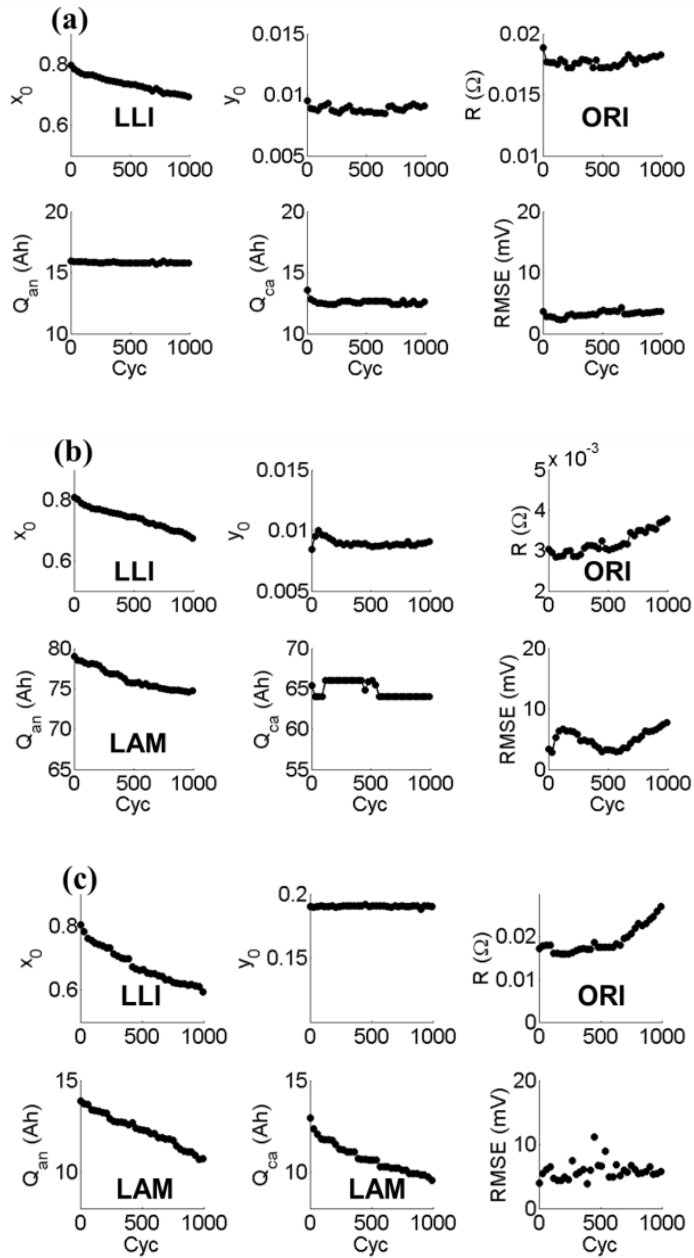

Figure 6 The identified parameters $\left\{Q_{c a}, Q_{a n}, y_{0}, x_{0}, R\right\}$ and the $R M S E$ of the MPM. (a) Battery A, (b) Battery B, (c) Battery C. 

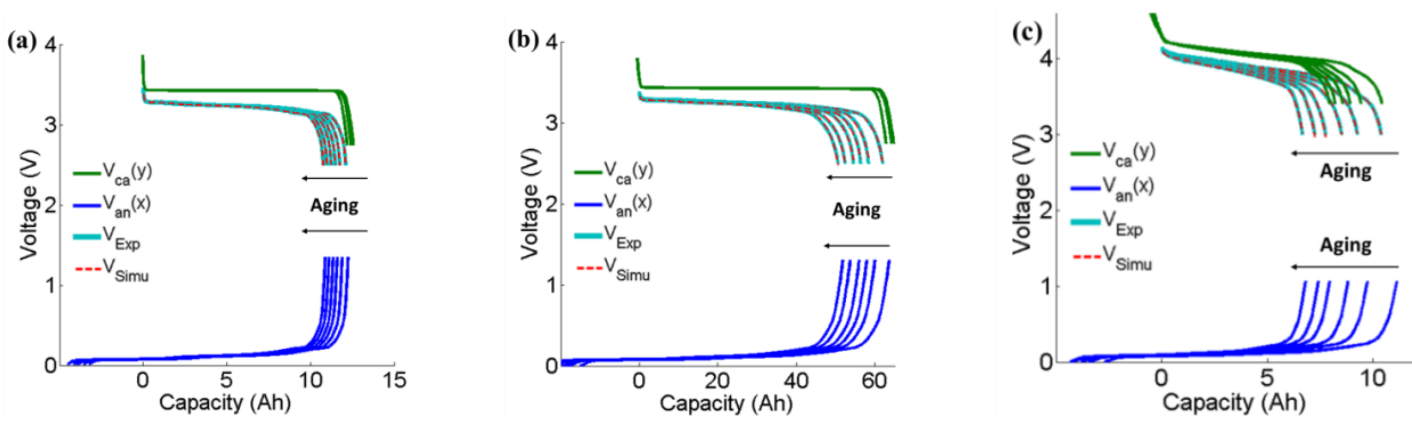

Figure 7 The good fit of the MPM with the experiment, using identified parameters. (a) Battery A, (b) Battery B, (c) Battery C.
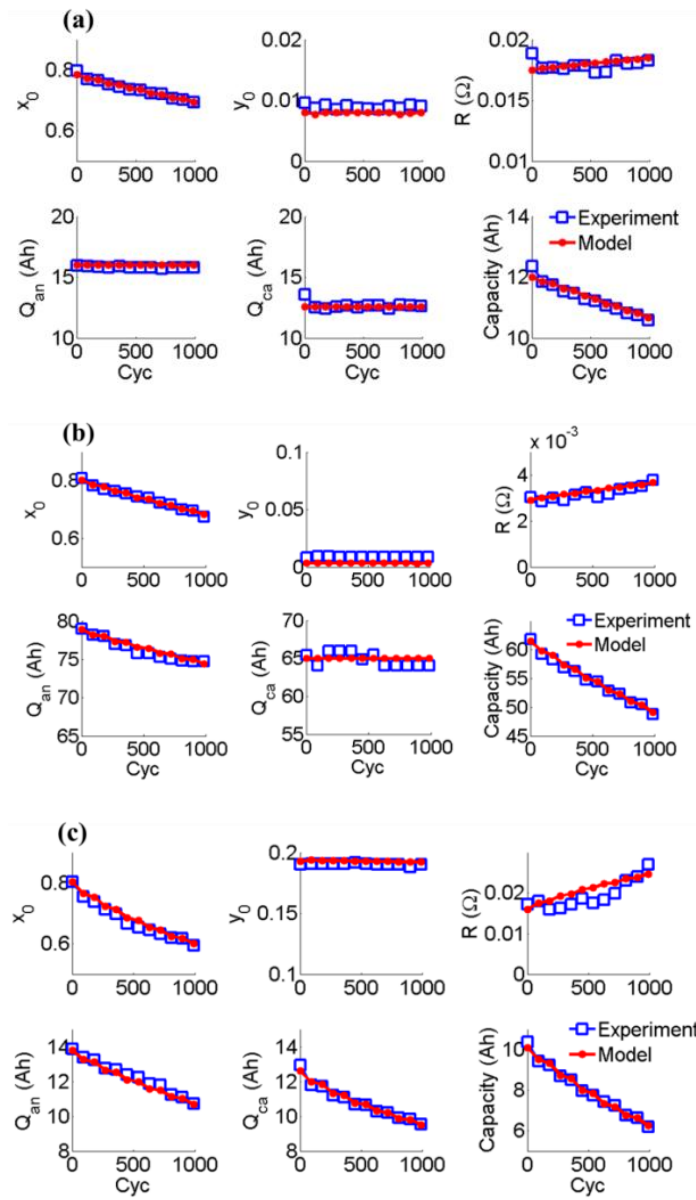

Figure 8 Comparison of the predicted parameters of the degradation model and the identified parameters in the MPM, (a) Battery A, (b) Battery B, (c) Battery C. 

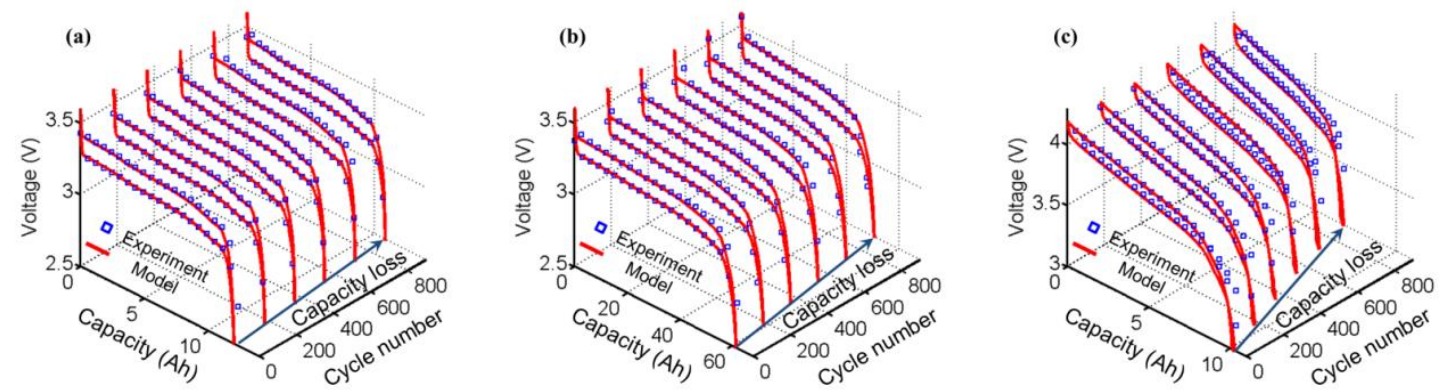

Figure 9 Comparison of the voltage curve of the degradation model and the experimental data under different $\mathrm{SOH}$, (a) Battery A, (b) Battery B, (c) Battery C.
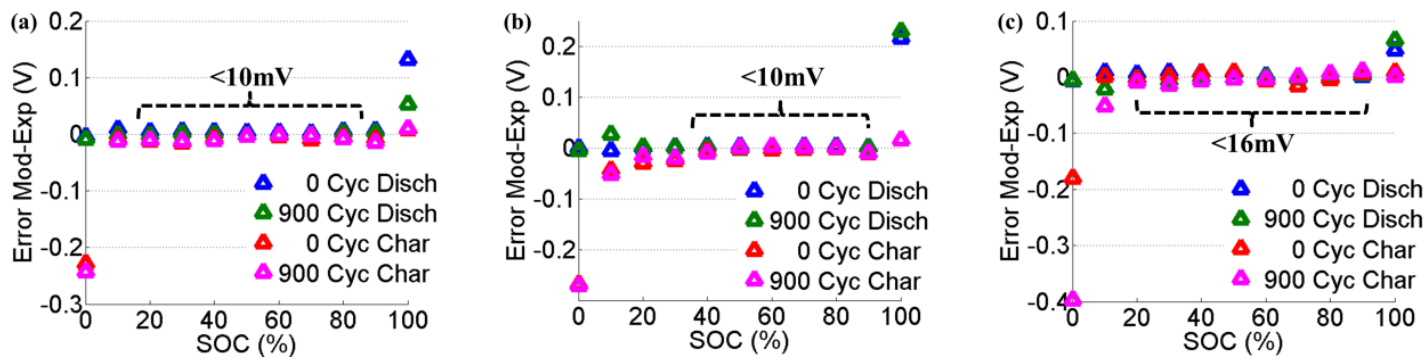

Figure 10 Prediction error of the degradation model. (a) Battery A, (b) Battery B, (c) Battery C.

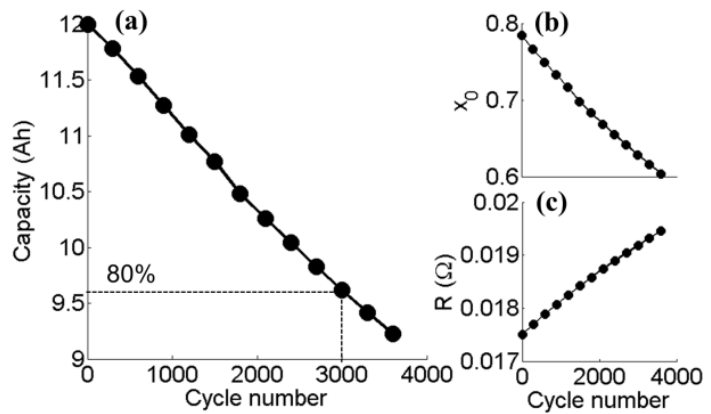

Figure 11 The capacity degradation of Battery A under $1 \mathrm{C}$ cycling at $25^{\circ} \mathrm{C}$.

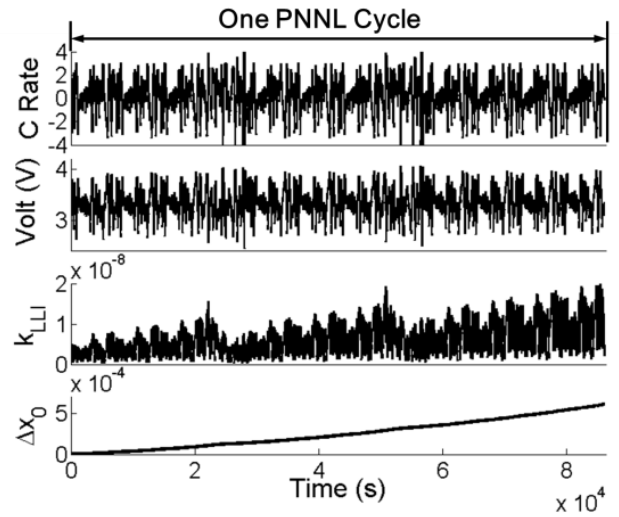

Figure 12 One PNNL cycle for the assessment of the ESS for grid use. 


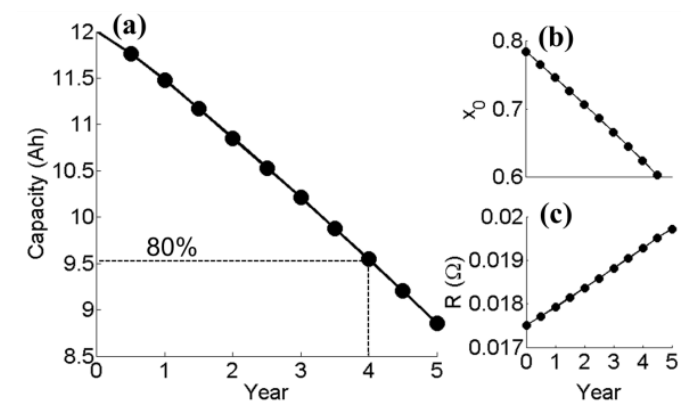

Figure 13 The predicted cycle life under PNNL cycle for the evaluation of grid use.

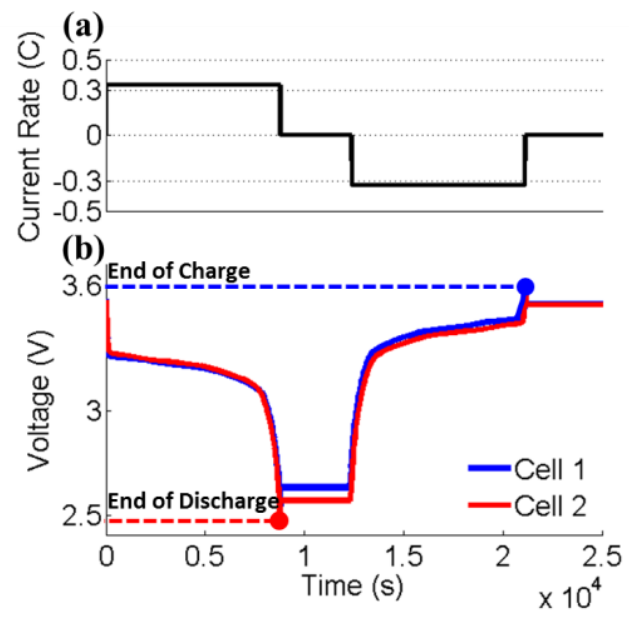

Figure 14 The voltage curve for each cell in the RPT test of the two-cell pack.
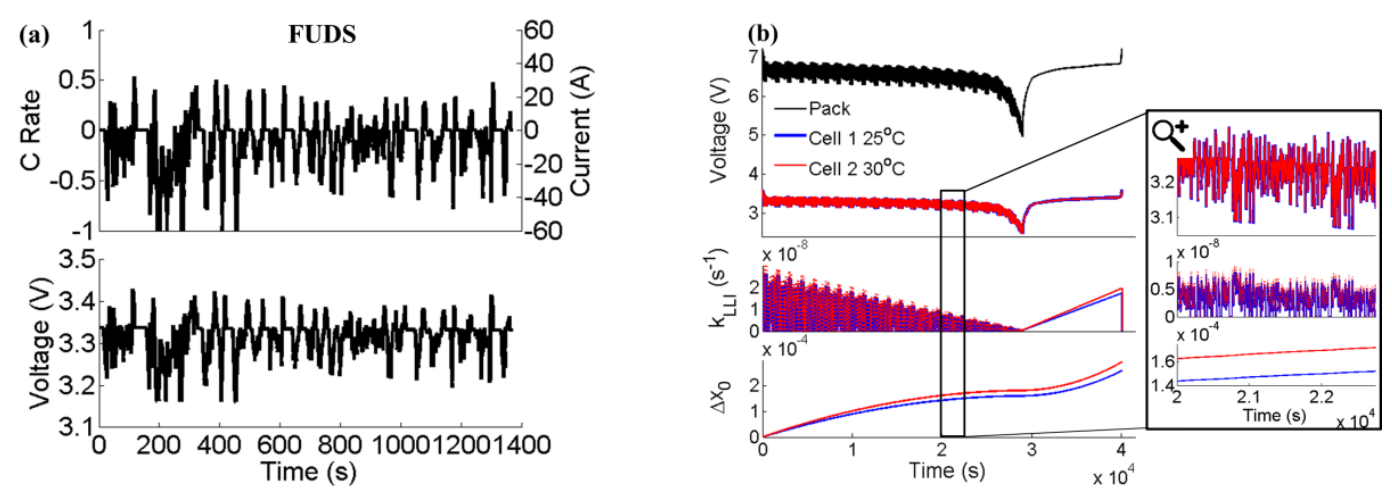

Figure 15 (a) The FUDS driving cycle; (b) One-day FUDS cycling and correlated LLI for the two-cell battery pack. 


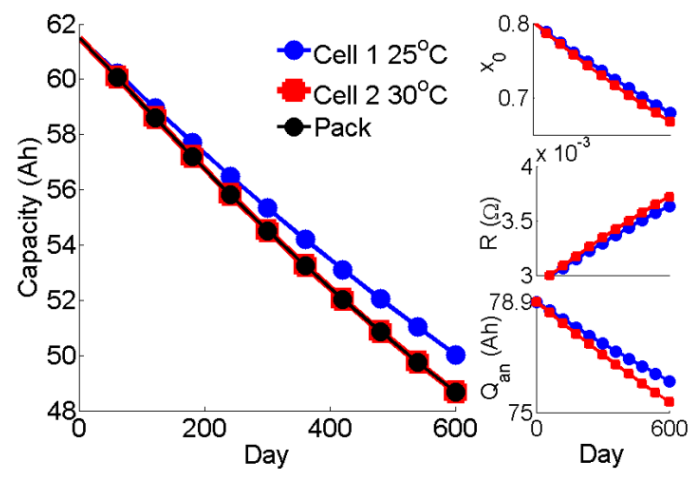

Figure 16 Cycle life prediction for the two-cell battery pack.

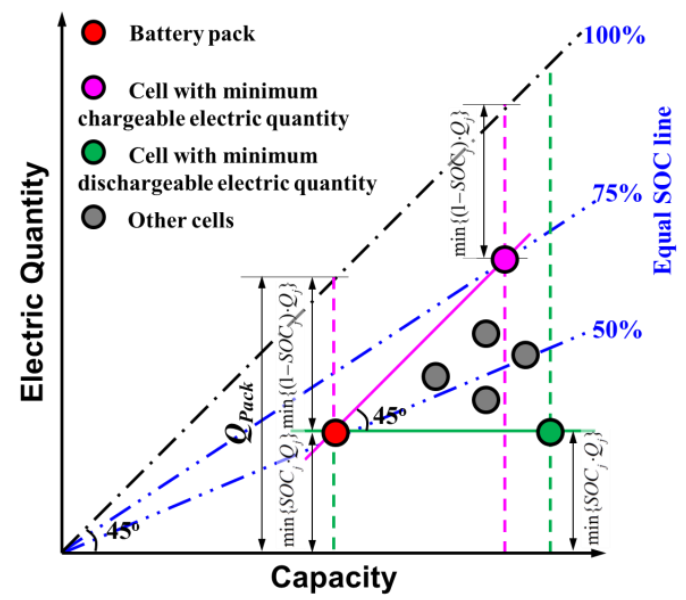

Figure 17 From cell capacity to the pack capacity: the $E-Q$ diagram as an illustration of Eqn. (17).

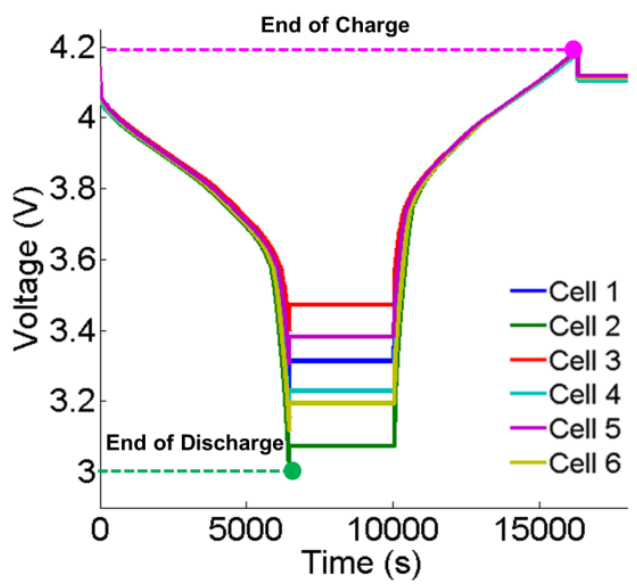

Figure 18 The voltage curve for each cell in the RPT test on the six-cell pack after 990 cycles. 

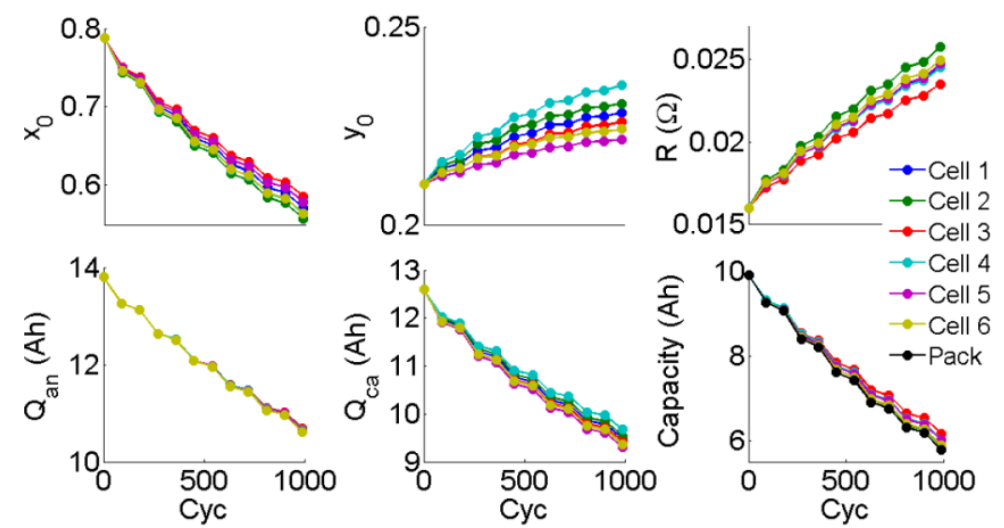

Figure 19 The evolution of the parameters in the MPM for each cell in the six-cell battery pack.

(a)

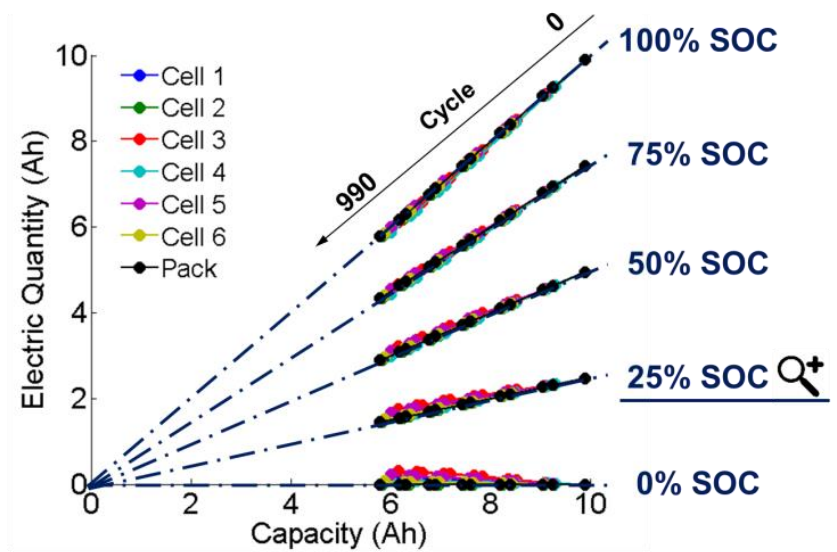

(b)

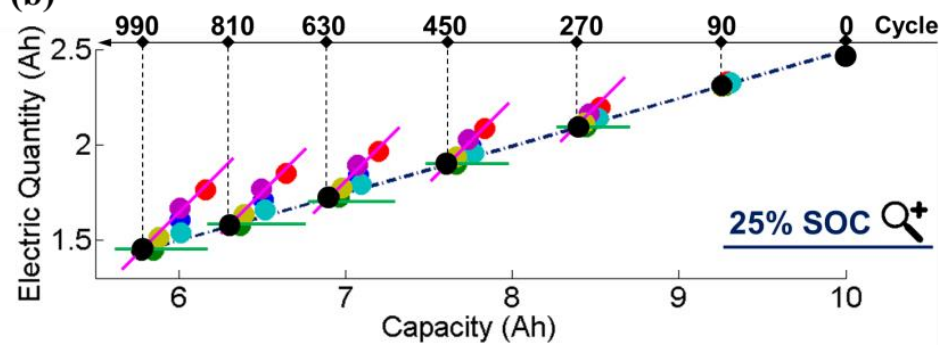

Figure 20 The evolution of the cell variations in the six-cell battery pack and the determination of the pack capacity. 
Table 1 Specifications of commercial batteries.

\begin{tabular}{ccccc}
\hline Battery & Cathode & Anode & Rated Capacity (Ah) & Voltage Range (V) \\
\hline A & LFP & G & 11 & $2.5-3.6$ \\
B & LFP & G & 60 & $2.5-3.6$ \\
C & LMO+NCM & G & 10 & $3.0-4.2$ \\
\hline
\end{tabular}

Table 2 The degradation mechanism of the batteries.

\begin{tabular}{ccccc}
\hline & \multicolumn{4}{c}{ Degradation mechanism } \\
\cline { 2 - 5 } Battery & & \multicolumn{2}{c}{ Anode } & Cathode \\
\cline { 2 - 5 } & ORI & LLI & LAM & LAM \\
\hline A & Y & Y & N & N \\
B & Y & Y & Y & N \\
C & Y & Y & Y & Y \\
\hline
\end{tabular}

Table 3 The values of $\xi(T)$ and $K_{\mathrm{SEI}}$ for different batteries in the model.

\begin{tabular}{|c|c|c|c|c|}
\hline \multirow{2}{*}{ Cell } & \multicolumn{3}{|c|}{$\xi(\boldsymbol{T})(\mathrm{K})$} & \multirow{2}{*}{$K_{S E I}$} \\
\hline & 278 & 298 & 318 & \\
\hline $\mathbf{A}$ & 1.6 & 1 & 0.8 & 0.011 \\
\hline B & 1.8 & 1 & 0.9 & 0.0055 \\
\hline C & 2 & 1 & 0.763 & 0.045 \\
\hline
\end{tabular}

Table 4 The subscript $\beta$ and the normalized concentration $c_{\beta}$ used in Eqn. (16).

\begin{tabular}{ccc}
\hline Degradation reaction & $\beta$ & $c_{\beta}$ \\
\hline LLI at anode & LLI & $x$ \\
LAM at anode & LAM,an & $c_{\text {LAM,an }}$ \\
LAM at cathode & LAM,ca & $c_{\text {LAM,ca }}$ \\
\hline
\end{tabular}

Table 5 Correlated parameters in Eqn. (16) for different degradation mechanisms.

\begin{tabular}{ccccccccc}
\hline \multirow{2}{*}{ Battery } & \multicolumn{2}{c}{$\begin{array}{c}\text { Degradation } \\
\text { mechanism }\end{array}$} & $A_{\beta} / \mathrm{s}^{-1}$ & $E_{a, \beta}$ & $n$ & $m$ & $K_{\beta}$ & $f(\beta)$ \\
\hline A & anode & LLI & $2.1 \times 10^{-5}$ & 37246 & 1 & 0.05 & 20000 & 1 \\
\hline \multirow{2}{*}{ B } & \multirow{2}{*}{ anode } & LLI & $3.561 \times 10^{-5}$ & 37246 & 1 & 0.05 & 20000 & 1 \\
& & LAM & $4.381 \times 10^{-3}$ & 43768 & 3 & 0.2 & 10000 & 1 \\
\hline \multirow{2}{*}{$\mathrm{C}$} & \multirow{2}{*}{ anode } & LLI & $1.236 \times 10^{-5}$ & 33146 & 1 & 0.05 & 20000 & 1 \\
& & LAM & $2.725 \times 10^{-4}$ & 41876 & 3 & 0.2 & 20000 & 1 \\
& cathode & LAM & $1.08 \times 10^{-3}$ & 44709 & 4 & 0.2 & 20000 & 1 \\
\hline
\end{tabular}


Table 6 The set of deviation in the degradation kinetics for the pack evolution simulation.

\begin{tabular}{cccc}
\hline Cell & $\boldsymbol{\Delta} \boldsymbol{k}_{\text {LLI }}$ & $\boldsymbol{\Delta} \boldsymbol{k}_{\text {LAM,ca }}$ & $\boldsymbol{\Delta} \boldsymbol{K}_{\text {SEI }}$ \\
\hline $\mathbf{1}$ & 0 & 0 & 0 \\
$\mathbf{2}$ & $+10 \%$ & $-5 \%$ & $+5 \%$ \\
$\mathbf{3}$ & $-10 \%$ & $+5 \%$ & $-5 \%$ \\
$\mathbf{4}$ & $+5 \%$ & $-10 \%$ & $-5 \%$ \\
$\mathbf{5}$ & $-5 \%$ & $+10 \%$ & $+5 \%$ \\
$\mathbf{6}$ & $+5 \%$ & $+5 \%$ & $0 \%$ \\
\hline
\end{tabular}

